\title{
Swinnerton-Dyer Type Congruences for certain Eisenstein series
}

\author{
Matthew Boylan
}

Jan 11, 2000.

\begin{abstract}
We consider a normalized Eisenstein series of weight $k$ on a congruence subgroup of type $\Gamma_{0}(N)$ with Nebentypus character $\chi$ which vanishes at all cusps of $\Gamma_{0}(N)$ inequivalent to the cusp at infinity. We determine conditions on $N, k, \chi$, and an ideal $\mathfrak{a}$ in certain number fields, under which their Fourier series are congruent to $1(\bmod \mathfrak{a})$.
\end{abstract}

\section{Introduction and Statement of Results}

If $k \geq 4$ is an even integer, then it is well-known [K, pg.111] that the normalized Eisenstein series given by

$$
E_{k}(z)=1-\frac{2 k}{B_{k}} \sum_{n \geq 1} \sigma_{k-1}(n) q^{n}
$$

is a modular form of weight $k$ with respect to $S L(2, \mathbb{Z})$, where $B_{k}$ is the $k$ th Bernoulli number, $q:=e^{2 \pi \mathrm{i} z}$, and $\sigma_{k}(n)$ is the function which sums the $k$ th powers of the positive divisors of $n$. Swinnerton-Dyer $[\mathbf{S w}-\mathbf{D}]$ showed that $E_{k}(z)$ satisfies the following congruence property:

Theorem (Swinnerton-Dyer). If $\ell \geq 5$ is a prime, then $E_{k}(z) \equiv 1(\bmod \ell)$ if and only if $k \equiv 0(\bmod \ell-1)$.

This follows from the Von Staudt-Claussen Theorem regarding the divisibility of the denominators of Bernoulli numbers.

Here we generalize Swinnerton-Dyer's result to certain Eisenstein series in spaces of modular forms of weight $k$ on a congruence subgroup of type $\Gamma_{0}(N)$ with Nebentypus character $\chi$. These spaces are denoted by $M_{k}\left(\Gamma_{0}(N), \chi\right)$. For background on integer weight modular forms, see $[\mathbf{K}]$. Following the methods in Sections 1-3 of Chapter VII of Schoeneberg's book, Elliptic Modular Functions, we develop the Fourier expansion at infinity of a normalized Eisenstein series $E_{N, k, \chi}(z) \in M_{k}\left(\Gamma_{0}(N), \chi\right)$ which vanishes at all cusps of $\Gamma_{0}(N)$ inequivalent to

1991 Mathematics Subject Classification. Primary 11F33.

Key words and phrases. Congruences for Eisenstein series. 
the cusp at infinity, and we state conditions on $N, k$, and $\chi$ guaranteeing the existence of these series. (Schoeneberg does this for Eisenstein series without character on an arbitrary subgroup of level $N$ ). Using these expansions, we obtain conditions on $N, k$, and $\chi$, and an ideal $\mathfrak{a}$ in certain number fields, under which $E_{N, k, \chi}(z) \equiv 1$ $(\bmod \mathfrak{a})$.

Theorem 1.1 lists formulas for $E_{N, k, \chi}(z)$ when they exist.

THEOREM 1.1. Suppose $\chi$ is a Dirichlet character with modulus $N$ and conductor $f$, and $\tau_{m}(d, \chi):=\sum_{h=1}^{m-1} \chi(h) \zeta_{m}^{d h}$, where $\zeta_{m}:=e^{\frac{2 \pi i}{m}}$. Suppose also that if $\chi$ is nontrivial and $N=1$ or 2 , then $k \geq 4$ is an even integer satisfying $\chi(-1)=(-1)^{k}$, and if $\chi$ is nontrivial and $N>2$, then $k \geq 3$ is an integer satisfying $\chi(-1)=(-1)^{k}$. Then the series $E_{N, k, \chi}(z)$ given by the following formulas are normalized modular forms in $M_{k}\left(\Gamma_{0}(N), \chi\right)$ which vanish at all cusps of $\Gamma_{0}(N)$ inequivalent to the cusp at infinity.

1. If $\chi$ is trivial and $N=1$, then for an even integer $k \geq 4$,

$$
E_{N, k, \chi}(z)=E_{k}(z)=1-\frac{2 k}{B_{k}} \sum_{n \geq 1} \sigma_{k-1}(n) q^{n}
$$

If $\chi$ is trivial and $N>1$, then for an even integer $k \geq 4$,

$$
E_{N, k, \chi}(z)=1-\frac{2 k \phi(N)}{N^{k} B_{k} \prod_{p \mid N}\left(1-\frac{1}{p^{k}}\right)} \sum_{n \geq 1}\left(\sum_{\substack{d \mid n \\ d>0}} d^{k-1} \frac{\mu(N / \operatorname{gcd}(d, N))}{\phi(N / \operatorname{gcd}(d, N))}\right) q^{n},
$$

where $\phi$ denotes Euler's phi function, and $\mu$ denotes the Möbius function.

2. If $\chi$ is nontrivial, then

$E_{N, k, \chi}(z)=1-\frac{k}{\left(\frac{N}{f}\right)^{k} \tau_{f}(1, \bar{\chi}) B_{k, \chi}} \sum_{n \geq 1}\left(\sum_{\substack{d \mid n \\ d>0}} d^{k-1}\left(\tau_{N}(d, \bar{\chi})+(-1)^{k} \tau_{N}(-d, \bar{\chi})\right)\right) q^{n}$, where $B_{k, \chi}$ is the generalized Bernoulli number associated to $\chi$.

3. If $\chi$ is nontrivial and primitive, then

$$
E_{N, k, \chi}(z)=1-\frac{2 k}{B_{k, \chi}} \sum_{n \geq 1}\left(\sum_{\substack{d \mid n \\ d>0}} \chi(d) d^{k-1}\right) q^{n}
$$

In what follows, let $K_{\chi}=\mathbb{Q}(\chi)$ denote the extension of $\mathbb{Q}$ obtained by adjoining the values of $\chi$, let $O_{K_{\chi}}$ denote the ring of integers of $K_{\chi}$, and denote by $O_{K_{\chi, N}}$ the ring of integers of $K_{\chi, N}=\mathbb{Q}\left(\chi, \zeta_{N}\right)$. We also define $\operatorname{ord}_{m}(n)$ to be the power of $m$ dividing $n$ if $m$ and $n$ are integers. If $\alpha=\frac{a}{b} \in \mathbb{Q}$, then $\operatorname{ord}_{m}(\alpha):=\operatorname{ord}_{m}(a)-$ $\operatorname{ord}_{m}(b)$. Theorems 1.2 and 1.3 generalize Swinnerton-Dyer's Theorem to the series (2) and (4) listed in Theorem 1.1. 
Theorem 1.2. Suppose that $\ell$ is an odd prime, $\chi$ is the trivial Dirichlet character modulo $N$, and $k \geq 4$ is an even integer. Then the following are true:

1. $E_{N, k, \chi}(z) \equiv 1(\bmod \ell)$ if and only if $k \equiv 0(\bmod \ell-1)$ and $N=\ell^{t}$ for some nonnegative integer $t$.

2. $E_{N, k, \chi}(z) \equiv 1(\bmod 2)$ if and only if $N=2^{a} p^{b}$, where $p$ is an odd prime satisfying $\operatorname{ord}_{2}\left(p^{k}-1\right)=1+\operatorname{ord}_{2}(k)$ and $a$ and $b$ are nonnegative integers.

Remark. Theorems 1.2.1 and 1.2.2 contain Swinnerton-Dyer's Theorem as a special case, the case where $N=1$.

TheOREM 1.3. Suppose that $\ell$ is an odd rational prime, $\mathfrak{a}$ is an ideal in $O_{K_{\chi}, N}$ with the property that $\mathfrak{a} \nmid(2)$, and $\chi$ is a nontrivial primitive Dirichlet character. Then for an integer $k \geq 3$ satisfying $\chi(-1)=(-1)^{k}$, we have:

1. If $N$ has at least two distinct prime divisors, then

$$
E_{N, k, \chi}(z) \not \equiv 1 \quad(\bmod \mathfrak{a}) .
$$

2. If $N=4$ and $\mathfrak{a} \nmid(4)$, then

$$
E_{4, k, \chi}(z) \not \equiv 1 \quad(\bmod \mathfrak{a}) .
$$

If $N=2^{t}$ for some integer $t \geq 3$, then

$$
E_{2^{t}, k, \chi}(z) \not \equiv 1 \quad(\bmod \mathfrak{a}) .
$$

3. If $N=\ell$, then

$$
E_{\ell, k, \chi}(z) \not \equiv 1 \quad(\bmod \mathfrak{a})
$$

unless there is a primitive root $g$ of $\mathbb{Z} / \ell \mathbb{Z}$ satisfying

$$
\mathfrak{p}=\operatorname{gcd}\left(\ell, 1-\chi(g) g^{k}\right) \neq(1),
$$

where $\mathfrak{p}$ is an ideal in $O_{K_{\chi}}$. In this case

$$
E_{\ell, k, \chi}(z) \equiv 1 \quad\left(\bmod \mathfrak{p}^{\operatorname{ord}_{\mathfrak{p}}(\ell)}\right) .
$$

4. If $N=\ell$ and $\chi=\left(\frac{\bullet}{\ell}\right)$, the Legendre symbol, then

$$
E_{\ell, k,\left(\frac{\bullet}{\ell}\right)} \equiv 1 \quad(\bmod \ell)
$$

if and only if $k \equiv \frac{\ell-1}{2}(\bmod \ell-1)$.

5. If $N=\ell^{t}$ for some integer $t \geq 2$, and if $\operatorname{gcd}\left(\ell, 1-\chi(g) g^{k}\right)=(1)$ for every primitive root $g$ of $\mathbb{Z} / \ell \mathbb{Z}$, then

$$
E_{\ell^{t}, k, \chi}(z) \not \equiv 1 \quad(\bmod \mathfrak{a}) .
$$

Note that if $\mathfrak{a}$ is an ideal in $O_{K_{\chi}, N}$ and if $j$ is a positive integer, then $E_{N, k, \chi}^{j}(z) \equiv$ $1(\bmod \mathfrak{a})$ whenever $E_{N, k, \chi}(z) \equiv 1(\bmod \mathfrak{a})$, where $E_{N, k, \chi}^{j}(z) \in M_{j k}\left(\Gamma_{0}(N), \chi\right)$. 


\section{Background on Schoeneberg's Eisenstein Series}

Before proceeding with the proof of Theorems 1.1-1.3, we describe the basic properties of Schoeneberg's primitive and reduced Eisenstein series, the building blocks for the Eisenstein series $E_{N, k, \chi}(z)$ that we construct. We keep the notation from Schoeneberg's book in what follows.

If $f: \mathbb{H} \mapsto \hat{\mathbb{C}}$, where $\mathbb{H}$ is the upper half plane and $\hat{\mathbb{C}}=\mathbb{C} \cup\{\infty\}$, and if $S=\left[\begin{array}{ll}a & b \\ c & d\end{array}\right] \in S L(2, \mathbb{Z})$, then $\left.f(z)\right|_{k} S:=(c z+d)^{-k} f(S z)$. Suppose that $N \geq 1$ and $k \geq 3$ are integers, and $\mathbf{m}=\left[\begin{array}{l}m_{1} \\ m_{2}\end{array}\right]$ and $\mathbf{a}=\left[\begin{array}{l}a_{1} \\ a_{2}\end{array}\right]$ are pairs of integers. Schoeneberg defines the inhomogenous Eisenstein series of weight $k$ and level $N$ as follows [Sc, pg.155, (2)]:

$$
G_{N, k, \mathbf{a}}(z)=\sum_{\substack{m_{1} \equiv a_{1}(\bmod N) \\ m_{2} \equiv a_{2}(\bmod N) \\ \mathbf{m} \neq \mathbf{0}}}\left(m_{1} z+m_{2}\right)^{-k},
$$

(In his notation, Schoeneberg refers to modular forms having dimension $-k<0$, rather than having weight $k>0$, which means the same. We prefer to use the term weight.) If $\operatorname{gcd}\left(a_{1}, a_{2}, N\right)=1$, then $G_{N, k, \mathbf{a}}(z)$ is called a primitive Eisenstein series.

The relevant facts about primitive Eisenstein series are these:

1. [Sc, pg.155, Thm.1] For all a, $G_{N, k, \mathbf{a}}(z) \in M_{k}(\Gamma(N))$.

2. $[$ Sc, pg.155, (3)] For all a,

$$
G_{N, k,-\mathbf{a}}(z)=(-1)^{k} G_{N, k, \mathbf{a}}(z) .
$$

3. $[\mathbf{S c}, \mathbf{p g} .155,(3)]$ If $\mathbf{a} \equiv \mathbf{a}_{1}(\bmod N)$, then

$$
G_{N, k, \mathbf{a}}(z)=G_{N, k, \mathbf{a}_{1}}(z) .
$$

4. [Sc, pg.156, (4)] If $A \in S L(2, \mathbb{Z})$, then

$$
\left.G_{N, k, \mathbf{a}}(z)\right|_{k} A=G_{N, k, A^{\prime} \mathbf{a}}(z),
$$

where $A^{\prime}$ is the transpose of $A$.

5. [Sc, pg.157, (5)] For integers $a$ and $b$, define

$$
\delta\left(\frac{a}{b}\right)= \begin{cases}1 & \text { if } b \mid a, \\ 0 & \text { if } b \nmid a .\end{cases}
$$

Then

$$
G_{N, k, \mathbf{a}}(z)=\sum_{\nu \geq 0} \alpha_{\nu}(N, k, \mathbf{a}) e^{\frac{2 \pi \mathrm{i} \nu}{N}}
$$

where

$$
\alpha_{0}(N, k, \mathbf{a})=\delta\left(\frac{a_{1}}{N}\right) \sum_{\substack{m_{2} \equiv a_{2}(\bmod N) \\ m_{2} \neq 0}} m_{2}^{-k}
$$


and for $\nu \geq 1$,

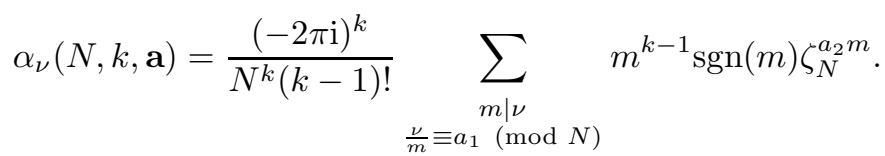

One may also define reduced Eisenstein series. If $\operatorname{gcd}\left(a_{1}, a_{2}, N\right)=1$, then these may be written $[\mathbf{S c}, \mathbf{p g} . \mathbf{1 5 8},(\mathbf{7})]$ :

$$
G_{N, k, \mathbf{a}}^{*}(z)=\sum_{\substack{\mathbf{m} \equiv \mathbf{a}(\bmod N) \\ \operatorname{gcd}\left(m_{1}, m_{2}\right)=1}}\left(m_{1} z+m_{2}\right)^{-k} .
$$

Facts 1-4 concerning primitive Eisenstein series also hold for reduced Eisenstein series. The reduced Eisenstein series are expressible as a linear combination of primitive Eisenstein series $[\mathbf{S c}, \mathbf{p g} . \mathbf{1 5 9},(\mathbf{9})]$ :

$$
G_{N, k, \mathbf{a}}^{*}(z)=\sum_{t(\bmod N)}\left(\sum_{d t \equiv 1(\bmod N)} \frac{\mu(d)}{d^{k}}\right) G_{N, k, t \mathbf{a}}(z) .
$$

We now proceed with the proof of Theorem 1.1

\section{The Proof of Theorem 1.1}

We note that $\Gamma_{0}(N)=\bigcup_{\nu=1}^{\mu_{1}} \Gamma(N) A_{\nu}$, where the coset representatives $A_{\nu}$ lie in the set

$$
\left\{\left[\begin{array}{ll}
\alpha_{\nu} & \beta_{\nu} \\
\gamma_{\nu} & \delta_{\nu}
\end{array}\right] \in S L(2, \mathbb{Z})\right\}
$$

where $\alpha_{\nu} \in(\mathbb{Z} / N \mathbb{Z})^{*}, \beta_{\nu} \in \mathbb{Z} / N \mathbb{Z}, \gamma_{\nu} \equiv 0(\bmod N), \delta_{\nu} \equiv \alpha_{\nu}^{-1}(\bmod N)$, and $\mu_{1}=\left[\Gamma_{0}(N): \Gamma(N)\right]$. We suppose that $\chi$ is a Dirichlet character with modulus $N$ and conductor $f$. As our goal is to construct modular forms for $M_{k}\left(\Gamma_{0}(N), \chi\right)$, we impose the condition that $k \geq 3$ is an integer with the property that

$$
\chi(-1)=(-1)^{k} \text {. }
$$

If $A=\left[\begin{array}{ll}a & b \\ c & d\end{array}\right] \in \Gamma_{0}(N)$, then the transformation law satisfied by modular forms $f(z) \in M_{k}\left(\Gamma_{0}(N), \chi\right)$ is given by:

$$
\left.f(z)\right|_{k} A=\chi(d) f(z) .
$$

An application of (14) using $A=-I \in \Gamma_{0}(N)$ shows that the spaces $M_{k}\left(\Gamma_{0}(N), \chi\right)$ contain only the modular form which is identically zero when (13) does not hold. We claim that

$$
G_{\Gamma_{0}(N), k, \chi,\left[\begin{array}{l}
0 \\
1
\end{array}\right]}^{*}(z)=\left.\sum_{\nu=1}^{\mu_{1}} \bar{\chi}\left(\delta_{\nu}\right) G_{N, k,\left[\begin{array}{l}
0 \\
1
\end{array}\right]}^{*}(z)\right|_{k} A_{\nu}
$$

is a modular form in $M_{k}\left(\Gamma_{0}(N), \chi\right)$ with the property that it vanishes at all cusps of $\Gamma_{0}(N)$ inequivalent to the cusp at infinity. 
Observing that $G_{\Gamma_{0}(N), k, \chi,\left[\begin{array}{l}0 \\ 1\end{array}\right]}^{*}(z)$ is a linear combination of reduced, and hence, by (11), primitive Eisenstein series, we impose the additional condition that $k \geq 4$ is an even integer when $N=1$ or 2 . When $N=1$ or 2 and $k \geq 3$ is an odd integer, it follows by (6) and (5) that

$$
\begin{aligned}
G_{N, k, \mathbf{a}}(z) & =G_{N, k,-\mathbf{a}}(z) \\
& =-G_{N, k, \mathbf{a}}(z) .
\end{aligned}
$$

This shows that $G_{N, k, \mathbf{a}}(z)=0$ in this case.

To verify that $G_{\Gamma_{0}(N), k, \chi,\left[\begin{array}{l}0 \\ 1\end{array}\right]}^{*}(z) \in M_{k}\left(\Gamma_{0}(N), \chi\right)$, we only need to show that it satisfies (14) since it clearly satisfies the remaining defining properties of a modular form in $M_{k}\left(\Gamma_{0}(N), \chi\right)$. If $A=\left[\begin{array}{ll}a & b \\ c & d\end{array}\right] \in \Gamma_{0}(N)$, then $A_{\nu} A=G_{\nu} A_{\nu^{\prime}}$ for some $G_{\nu} \in \Gamma(N)$, and for some $\nu^{\prime}$ uniquely determined by $\nu$ which runs through $\left\{1, \ldots, \mu_{1}\right\}$ as $\nu$ does. Moreover, $A_{\nu^{\prime}}=\left[\begin{array}{cc}\alpha_{\nu^{\prime}} & \beta_{\nu^{\prime}} \\ \gamma_{\nu^{\prime}} & \delta_{\nu^{\prime}}\end{array}\right]$, with $\delta_{\nu} \equiv d^{-1} \delta_{\nu^{\prime}}(\bmod N)$. Therefore,

$$
\begin{aligned}
\left.G_{\Gamma_{0}(N), k, \chi,\left[\begin{array}{l}
0 \\
1
\end{array}\right]}^{*}(z)\right|_{k} A & =\left.\sum_{\nu=1}^{\mu_{1}} \bar{\chi}\left(\delta_{\nu}\right) G_{N, k,\left[\begin{array}{l}
0 \\
1
\end{array}\right]}^{*}(z)\right|_{k} A_{\nu} A \\
& =\sum_{\nu^{\prime}=1}^{\mu_{1}} \bar{\chi}\left(d^{-1} \delta_{\nu^{\prime}}\right) G_{N, k,[}^{*}\left[\begin{array}{l}
0 \\
1
\end{array}\right] \\
& =\left.\chi(d)\right|_{k} G_{\nu} A_{\nu^{\prime}} \\
& =\chi(d) G_{\nu^{\prime}=1}^{\mu_{1}} \bar{\chi}\left(\delta_{\nu^{\prime}}\right) G_{N, k, k, k,}^{*}\left[\begin{array}{l}
0 \\
1
\end{array}\right] \\
& \left.(z)\right|_{k} A_{\nu^{\prime}}
\end{aligned}
$$

so $G_{\Gamma_{0}(N), k, \chi,\left[\begin{array}{l}0 \\ 1\end{array}\right]}^{*}(z) \in M_{k}\left(\Gamma_{0}(N), \chi\right)$.

Next, we calculate the value of $\left.G_{\Gamma_{0}(N), k, \chi}^{*},\left[\begin{array}{l}0 \\ 1\end{array}\right]\right)$ at an arbitrary cusp $\frac{-d}{c}$. To do this, we form $A=\left[\begin{array}{ll}a & b \\ c & d\end{array}\right] \in S L(2, \mathbb{Z})$, and consider $\left.G_{\Gamma_{0}(N), k, \chi,}^{*}\left[\begin{array}{l}0 \\ 1\end{array}\right]\right)\left.(z)\right|_{k} A^{-1}=$ $\sum_{n \geq 0} r(n) q^{\frac{n}{N}}$. The value of $G_{\Gamma_{0}(N), k, \chi,[}^{*}\left[\begin{array}{l}0 \\ 1\end{array}\right](z)$ at $\frac{-d}{c}$ is $r(0)$. The first step in the calculation is to simplify $\left.G_{\Gamma_{0}(N), k, \chi,\left[\begin{array}{l}0 \\ 1\end{array}\right]}^{*}(z)\right|_{k} A^{-1}$ using (7) twice and (12):

$$
\begin{aligned}
& \left.G_{\Gamma_{0}(N), k, \chi,\left[\begin{array}{l}
0 \\
1
\end{array}\right]}^{*}(z)\right|_{k} A^{-1}=\left.\sum_{\nu=1}^{\mu_{1}} \bar{\chi}\left(\delta_{\nu}\right) G_{N, k,\left[\begin{array}{l}
0 \\
1
\end{array}\right]}^{*}(z)\right|_{k} A_{\nu} A^{-1} \\
& =\sum_{\nu=1}^{\mu_{1}} \bar{\chi}\left(\delta_{\nu}\right) G_{N, k,[}^{*}\left[\begin{array}{l}
\gamma_{\nu} \\
\delta_{\nu}
\end{array}\right]\left[\left.(z)\right|_{k} A^{-1}\right. \\
& =\left.N \sum_{h \in(\mathbb{Z} / N \mathbb{Z})^{*}} \bar{\chi}(h) G_{N, k,[}^{*}\left[\begin{array}{l}
0 \\
h
\end{array}\right](z)\right|_{k} A^{-1} \\
& =N \sum_{h \in(\mathbb{Z} / N \mathbb{Z})^{*}} \bar{\chi}(h) G_{N, k,\left[\begin{array}{c}
-c h \\
a h
\end{array}\right]}(z) .
\end{aligned}
$$


In view of (15), we simplify $G_{N, k,\left[\begin{array}{c}-c h \\ a h\end{array}\right]}(z)$ using (11):

$$
\begin{aligned}
G_{N, k,\left[\begin{array}{c}
-c h \\
a h
\end{array}\right]}^{*}(z) & =\sum_{\nu \geq 0} \alpha_{\nu}^{*}\left(N, k,\left[\begin{array}{c}
-c h \\
a h
\end{array}\right]\right) e^{\frac{2 \pi \mathrm{i} \nu z}{N}} \\
& =\sum_{t \in(\mathbb{Z} / N \mathbb{Z})^{*}}\left(\sum_{d t \equiv 1} \frac{\mu(d)}{\left.\bmod _{d>0}\right)}\right) G_{N, k,\left[\begin{array}{c}
-t c h \\
t a h
\end{array}\right]}(z) .
\end{aligned}
$$

We follow Schoeneberg's computation of $\alpha_{0}^{*}\left(N, k,\left[\begin{array}{c}-c h \\ a h\end{array}\right]\right)$ and note that the first equality is obtained by applying (9) and (16) [Sc, pg.160]:

$$
\begin{aligned}
& \alpha_{0}^{*}\left(N, k,\left[\begin{array}{c}
-c h \\
a h
\end{array}\right]\right)=\sum_{t \in(\mathbb{Z} / N \mathbb{Z})^{*}}\left(\sum_{d t \equiv 1} \frac{\mu(d)}{\bmod _{d>0}}\right) \delta\left(\frac{-t c h}{N}\right) \sum_{\substack{m \equiv t a h(\bmod N) \\
m \neq 0}} m^{-k} \\
& =\delta\left(\frac{-c h}{N}\right) \sum_{d>0} \sum_{\substack{m d \equiv a h(\bmod N) \\
m \neq 0}} \frac{\mu(d)}{(d m)^{k}} \\
& =\delta\left(\frac{-c h}{N}\right) \sum_{\substack{m \equiv a h(\bmod N) \\
m \neq 0}} m^{-k} \sum_{\substack{d \mid m \\
d>0}} \mu(d)
\end{aligned}
$$

Observe that since

$$
\sum_{\substack{d \mid m \\ d>0}} \mu(d)= \begin{cases}1 & \text { if } m=1, \text { or }-1 \\ 0 & \text { if } m \neq 1, \text { or }-1\end{cases}
$$

it follows that $\alpha_{0}^{*}\left(N, k,\left[\begin{array}{c}-c h \\ a h\end{array}\right]\right) \neq 0$ if and only if $c \equiv 0(\bmod N)$ and $d \equiv h$ $(\bmod N)$, i.e., if and only if $\frac{-d}{c}$ is $\Gamma(N)$-equivalent to the cusp at infinity. Continuing our calculation, we now have

$$
r(0)=N \sum_{h \in(\mathbb{Z} / N \mathbb{Z})^{*}} \bar{\chi}(h) \alpha_{0}^{*}\left(N, k,\left[\begin{array}{c}
-c h \\
a h
\end{array}\right]\right)
$$

by (15). Therefore, $r(0)=0$ at all cusps of $\Gamma(N)$ inequivalent to the cusp at infinity, and hence, at all cusps of $\Gamma_{0}(N)$ inequivalent to the cusp at infinity since $\Gamma(N) \subset \Gamma_{0}(N)$.

It remains to show that the value of $G_{\Gamma_{0}(N), k, \chi,\left[\begin{array}{l}0 \\ 1\end{array}\right]}^{*}(z)$ at the cusp at infinity is nonzero. Combining the previous facts given by (17), (19), (18), and (13), we calculate:

$$
\begin{aligned}
& r(0)=N \sum_{h \in(\mathbb{Z} / N \mathbb{Z})^{*}} \bar{\chi}(h) \sum_{\substack{m \equiv h(\bmod N) \\
m \neq 0}} m^{-k} \sum_{\substack{d \mid m \\
d>0}} \mu(d) \\
& =N\left(\bar{\chi}(1)(1)^{k}+\bar{\chi}(-1)(-1)^{k}\right) \\
& =2 N \text {. }
\end{aligned}
$$


This proves that $G_{\Gamma_{0}(N), k, \chi,\left[\begin{array}{l}0 \\ 1\end{array}\right]}^{*}(z)$ satisfies the cusp conditions stated in Theorem 1.1 .

We now develop the normalized Fourier expansion of $G_{\Gamma_{0}(N), k, \chi,\left[\begin{array}{l}0 \\ 1\end{array}\right]}^{*}(z)$ at infinity. Recall that $k$ is even when $N=1$ or 2 . Letting $s_{t}:=\sum_{d t \equiv 1} \frac{\mu(d)}{\bmod _{d>0}} \frac{\mu}{d^{k}}$ for $t \in(\mathbb{Z} / N \mathbb{Z})^{*}$, we simplify $G_{\Gamma_{0}(N), k, \chi,\left[\begin{array}{l}0 \\ 1\end{array}\right]}^{*}(z)$ using $(15)$ and (11):

$$
\begin{aligned}
G_{\Gamma_{0}(N), k, \chi,[}^{*}\left[\begin{array}{l}
0 \\
1
\end{array}\right] & (z)=N \sum_{h \in(\mathbb{Z} / N \mathbb{Z})^{*}} \bar{\chi}(h) G_{N, k,\left[\begin{array}{l}
0 \\
h
\end{array}\right]}^{*}(z) \\
& =N \sum_{h \in(\mathbb{Z} / N \mathbb{Z})^{*}} \bar{\chi}(h) \sum_{t \in(\mathbb{Z} / N \mathbb{Z})^{*}} s_{t} G_{N, k,\left[\begin{array}{c}
0 \\
t h
\end{array}\right]}(z) .
\end{aligned}
$$

Letting $c(N):=N \sum_{t \in(\mathbb{Z} / N \mathbb{Z})^{*}} s_{t} \chi(t)$, a constant dependent only on $N$, and making the change of variable $j=t h$, we obtain

$$
G_{\Gamma_{0}(N), k, \chi,\left[\begin{array}{l}
0 \\
1
\end{array}\right]}^{*}(z)=c(N) \sum_{j \in(\mathbb{Z} / N \mathbb{Z})^{*}} \bar{\chi}(j) G_{N, k,\left[\begin{array}{l}
0 \\
j
\end{array}\right]}(z) .
$$

Substituting (9) and (10) in (21), we have

$$
\begin{aligned}
& G_{\Gamma_{0}(N), k, \chi,\left[\begin{array}{l}
0 \\
1
\end{array}\right]}^{*}(z)=c(N) \sum_{j \in(\mathbb{Z} / N \mathbb{Z})^{*}} \bar{\chi}(j) \times \\
& \left(\sum_{\substack{m \equiv j(\bmod N) \\
m \neq 0}} m^{-k}+\frac{(-2 \pi \mathrm{i})^{k}}{N^{k}(k-1) !} \sum_{n \geq 1}\left(\sum_{\substack{d \mid n \\
d}} d^{k-1} \operatorname{sgn}(d) \zeta_{N}^{d j}\right) q^{\frac{n}{N}}\right) .
\end{aligned}
$$

Using (22) and (13), observe that the constant term $r(0)$ may also be expressed as

$$
\begin{aligned}
& r(0)=c(N) \sum_{j \in(\mathbb{Z} / N \mathbb{Z})^{*}} \bar{\chi}(j) \sum_{m \equiv j(\bmod N)} m^{-k} \\
& =c(N) \sum_{m \geq 1} \bar{\chi}(m) m^{-k}\left(1+\bar{\chi}(-1)(-1)^{k}\right) \\
& =2 c(N) L(k, \bar{\chi}),
\end{aligned}
$$


where $L(k, \chi)$ is the Dirichlet $L$-function associated to $\chi$. Substituting (23) in (22) gives us

$$
\begin{aligned}
& G_{\Gamma_{0}(N), k, \chi,\left[\begin{array}{l}
0 \\
1
\end{array}\right]}^{*}(z)=c(N) \times \\
& \left(2 L(k, \bar{\chi})+\frac{(-2 \pi \mathrm{i})^{k}}{N^{k}(k-1) !} \sum_{n \geq 1}\left(\sum_{j \in(\mathbb{Z} / N \mathbb{Z})^{*}} \bar{\chi}(j) \sum_{\substack{d \mid n \\
\frac{n}{d} \equiv 0}(\bmod N)} d^{k-1} \operatorname{sgn}(d) \zeta_{N}^{d j}\right) q^{\frac{n}{N}}\right) .
\end{aligned}
$$

It is clear from (20) and (23) that $c(N) \neq 0$. Therefore, we can define

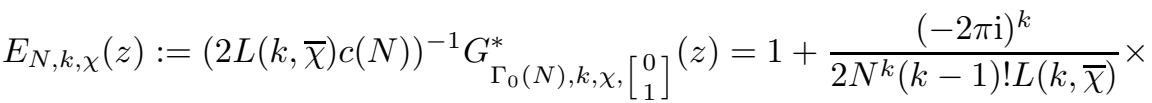

$$
\begin{aligned}
& \sum_{n \geq 1}\left(\sum_{j \in(\mathbb{Z} / N \mathbb{Z})^{*}} \bar{\chi}(j) \sum_{\substack{d \mid n \\
\frac{n}{d} \equiv 0(\bmod N)}} d^{k-1} \operatorname{sgn}(d) \zeta_{N}^{d j}\right) q^{\frac{n}{N}} \in M_{k}\left(\Gamma_{0}(N), \chi\right) .
\end{aligned}
$$

Noting that

$$
\begin{aligned}
\sum_{j \in(\mathbb{Z} / N \mathbb{Z})^{*}} \bar{\chi}(j) \sum_{d \mid n} d^{k-1} \operatorname{sgn}(d) \zeta_{N}^{d j} & =\sum_{j \in(\mathbb{Z} / N \mathbb{Z})^{*}} \bar{\chi}(j) \sum_{\substack{d \mid n \\
d>0}} d^{k-1}\left(\zeta_{N}^{d j}+(-1)^{k} \zeta_{N}^{-d j}\right) \\
& =\sum_{\substack{d \mid n \\
d>0}} d^{k-1}\left(\tau_{N}(d, \bar{\chi})+(-1)^{k} \tau_{N}(-d, \bar{\chi})\right),
\end{aligned}
$$

and that the condition $\frac{n}{d} \equiv 0(\bmod N)$ allows us to make the change of variable $n \rightarrow n N$, our formula becomes:

$$
E_{N, k, \chi}(z)=1+\frac{(-2 \pi \mathrm{i})^{k}}{2 N^{k}(k-1) ! L(k, \bar{\chi})} \sum_{n \geq 1}\left(\sum_{\substack{d \mid n \\ d>0}} d^{k-1}\left(\tau_{N}(d, \bar{\chi})+(-1)^{k} \tau_{N}(-d, \bar{\chi})\right)\right) q^{n} .
$$

We now simplify $E_{N, k, \chi}(z)$ in the three cases specified in Theorem 1.1 using certain well-known facts. If $\chi$ is the trivial character with modulus $N$, then $\bar{\chi}=\chi$ and $k$ is even. We use the following facts to obtain formulas (1) and (2):

1. [Ir-R, Thm.2, pg.231] If $k$ is a positive even integer, then

$$
2 \zeta(k)=\frac{(-1)^{\frac{k}{2}+1}(2 \pi)^{k} B_{k}}{k !},
$$

where $\zeta(s)$ is the Riemann zeta function.

2. [Ir-R, pg.255] If $\chi$ is trivial, then

$$
L(k, \chi)= \begin{cases}\zeta(k) & \text { if } N=1, \\ \zeta(k) \prod_{p \mid N}\left(1-\frac{1}{p^{k}}\right) & \text { if } N>1 .\end{cases}
$$


3. $[\mathbf{A}, \mathbf{p g . 1 6 4}]$ If $\chi$ is trivial, then

$$
\begin{aligned}
\tau_{N}(d, \chi) & =\tau_{N}(-d, \chi) \\
& =\frac{\phi(N) \mu(N / \operatorname{gcd}(d, N))}{\phi(N / \operatorname{gcd}(d, N))} .
\end{aligned}
$$

When $\chi$ is a nontrivial character with modulus $N$ and conductor $f$, we use a different set of facts to produce the formula (3):

1. $[\mathbf{I r}-\mathbf{R}$, Prop.16.6.2 $]$ If $\chi$ is nontrivial and $k$ is a positive integer, then

$$
L(1-k, \chi)=-\frac{B_{k, \chi}}{k} .
$$

2. If $k$ is a positive integer, then

$$
\Gamma(k)=(k-1) !
$$

where $\Gamma(s)$ is the classical $\Gamma$-function.

3. If $\chi$ is nontrivial and $k$ is a positive integer, and if we define

$$
\delta_{\chi}:= \begin{cases}1 & \text { if } \chi(-1)=-1, \\ 0 & \text { if } \chi(-1)=1,\end{cases}
$$

then the functional equation for $L(k, \chi)$ is $[\mathbf{I w}, \mathbf{C h . 1}$, Sec.1.2]:

$$
L(k, \chi)=\frac{\tau_{f}(1, \chi)}{2 \mathrm{i}^{\delta_{\chi}}}\left(\frac{2 \pi}{f}\right)^{k} \frac{L(1-k, \bar{\chi})}{\Gamma(k) \cos \left(\frac{\pi\left(k-\delta_{\chi}\right)}{2}\right)} .
$$

If $\chi$ is a nontrivial primitive character with modulus $N$, we know the additional fact $[\mathbf{A}, \mathbf{T h m} . \mathbf{8 . 1 5}]$ :

$$
\tau_{N}(d, \chi)=\chi(d) \tau_{N}(1, \chi)
$$

which we substitute in (3) to obtain (4). This finishes the proof of Theorem 1.1.

\section{The Proof of Theorem 1.2}

The proof of Theorem 1.2 relies on some well-known facts about the ordinary Bernoulli numbers, $B_{k}$ :

Theorem 4.1 (Von Staudt-Claussen) [Ir-R, Thm.3, pg.233]. Suppose that $\ell$ is a prime and $k$ is a positive even integer. If $\ell-1 \nmid k$, then $\operatorname{ord}_{\ell}\left(B_{k}\right) \geq 0$, and if $\ell-1 \mid k$, then $\operatorname{ord}_{\ell}\left(B_{k}\right)=-1$.

TheOREm 4.2 [Ir-R, Thm.15.2.4]. Suppose that $\ell$ is a prime and $k$ is a positive even integer. If $\ell-1 \nmid k$, then $\operatorname{ord}_{\ell}\left(\frac{B_{k}}{k}\right) \geq 0$.

In the case where $\chi$ is the trivial character modulo 1 and $\ell$ is a rational prime, the desired result follows by applying Theorem 4.1 to formula (1). This is Swinnerton-Dyer's Theorem. 
Therefore, we consider the cases in which $N>1$. We let $E_{N, k, \chi}(z)=\sum_{n \geq 0} a(n) q^{n}$ and $c_{N}:=\frac{N}{\prod_{p \mid N} p}$. To start, we simplify the coefficient $a\left(c_{N}\right)$ :

$$
a\left(c_{N}\right)=\frac{-2 k \phi(N)}{N^{k} B_{k} \prod_{p \mid N}\left(1-\frac{1}{p^{k}}\right)} \sum_{d \mid} d_{\prod_{p \mid N^{p}}}^{k-1} \frac{\mu(N / \operatorname{gcd}(d, N))}{\phi(N / \operatorname{gcd}(d, N)} .
$$

Since

$$
\mu(N / \operatorname{gcd}(d, N))= \begin{cases}(-1)^{\omega(N)} & \text { if } d=N / \prod_{p \mid N} p, \\ 0 & \text { if } d<N / \prod_{p \mid N} p,\end{cases}
$$

where $\omega(N)$ is the number of distinct prime divisors of $N$, it follows that

$$
a\left(c_{N}\right)=\frac{(-1)^{\omega(N)+1} 2 k}{B_{k} \prod_{p \mid N}\left(p^{k}-1\right)} .
$$

Note that

$$
\operatorname{ord}_{\ell}\left(a\left(c_{N}\right)\right)=\operatorname{ord}_{\ell}(2)+\operatorname{ord}_{\ell}(k)-\operatorname{ord}_{\ell}\left(B_{k}\right)-\sum_{p \mid N} \operatorname{ord}_{\ell}\left(p^{k}-1\right)
$$

for a given prime $\ell$. Assuming for now that $\ell$ is odd, we analyze $a\left(c_{N}\right)(\bmod \ell)$ in several cases.

CASE $1 . \ell-1 \nmid k$.

$\operatorname{ord}_{\ell}\left(\frac{B_{k}}{k}\right) \geq 0$ by Theorem 4.2 , so $\operatorname{ord}_{\ell}\left(a\left(c_{N}\right)\right) \leq 0$ by $(24)$, and hence, $E_{N, k, \chi}(z) \not \equiv$ $1(\bmod \ell)$.

Cases $2 \mathrm{a}$ and $2 \mathrm{~b}$ concern the situation where $\ell-1 \mid k$. In this situation, Theorem 4.1 implies that $\operatorname{ord}_{\ell}\left(B_{k}\right)=-1$. We suppose that $\operatorname{ord}_{\ell}(k)=j$. Then

$$
k=\ell^{j}(\ell-1) m=\phi\left(\ell^{j+1}\right) m
$$

for some positive integer $m$ coprime to $\ell$.

CASE 2 A. $\ell-1 \mid k$ and $\ell \nmid N$.

$\operatorname{gcd}(p, \ell)=1$ for every prime $p \mid N$ since $\ell \nmid N$, so $p^{k} \equiv 1\left(\bmod \ell^{j+1}\right)$ by $(25)$. Using (24) we have

$$
\operatorname{ord}_{\ell}\left(a\left(c_{N}\right)\right) \leq(j+1)(1-\omega(N)) .
$$

$\omega(N) \geq 1$ since $N>1$, so $\operatorname{ord}_{\ell}\left(a\left(c_{N}\right)\right) \leq 0$ in this case. Consequently, $E_{N, k, \chi}(z) \not \equiv 1$ $(\bmod \ell)$.

CASE 2B. $\ell-1 \mid k$ and $\ell \mid N$.

Using (24) and (25) again, we have

$$
\operatorname{ord}_{\ell}\left(a\left(c_{N}\right)\right) \leq(j+1)(2-\omega(N)) .
$$

It follows that if $N$ is not a positive power of $\ell$, then $\operatorname{ord}_{\ell}\left(a\left(c_{N}\right)\right) \leq 0$, in which case $E_{N, k, \chi}(z) \not \equiv 1(\bmod \ell)$. 
Therefore, in the case where $\ell$ is an odd prime we know the following:

$E_{N, k, \chi}(z) \not \equiv 1(\bmod \ell)$ if $k \not \equiv 0(\bmod \ell-1)$ or if $N \neq \ell^{t}$ for all positive integers $t$. We now prove the converse.

For an odd prime $\ell$ and a positive integer $t$, we simplify $E_{\ell^{t}, k, \chi}(z)$ by first observing that

$$
\begin{aligned}
\frac{\phi\left(\ell^{t}\right) \mu\left(\ell^{t} / \operatorname{gcd}\left(d, \ell^{t}\right)\right)}{\phi\left(\ell^{t} / \operatorname{gcd}\left(d, \ell^{t}\right)\right.} & = \begin{cases}\ell^{t-1}(\ell-1) & \text { if } \operatorname{ord}_{\ell}(d)=t, \\
-\ell^{t-1} & \text { if } \operatorname{ord}_{\ell}(d)=t-1, \\
0 & \text { if } \operatorname{ord}_{\ell}(d) \leq t-2 .\end{cases} \\
& =\ell^{t-1}\left(\delta\left(\frac{d}{\ell^{t}}\right) \ell-\delta\left(\frac{d}{\ell^{t-1}}\right)\right),
\end{aligned}
$$

using the notation defined by (8). After substituting (26) in (2), we obtain

$$
\begin{aligned}
& E_{\ell^{t}, k, \chi}(z)=1-\frac{2 k \ell^{t-1}}{\ell^{t k} B_{k}\left(1-\frac{1}{\ell^{k}}\right)} \times \\
& \left(\sum_{n \geq 1}\left(\sum_{\substack{d \mid n \\
d>0}} d^{k-1} \delta\left(\frac{d}{\ell^{t}}\right) \ell\right) q^{n}-\sum_{n \geq 1}\left(\sum_{\substack{d \mid n \\
d>0}} d^{k-1} \delta\left(\frac{d}{\ell^{t-1}}\right)\right) q^{n}\right) .
\end{aligned}
$$

Making the change of variables $d \rightarrow d \ell^{t}$ gives us

$$
\begin{aligned}
\sum_{n \geq 1}\left(\sum_{\substack{d \mid n \\
d>0}} d^{k-1} \delta\left(\frac{d}{\ell^{t}}\right) \ell\right) q^{n} & =\ell^{t(k-1)+1} \sum_{n \geq 1}\left(\sum_{\substack{d \mid \frac{n}{\ell^{t}} \\
d>0}} d^{k-1}\right) q^{n} \\
& =\ell^{t(k-1)+1} \sum_{n \geq 1} \sigma_{k-1}\left(\frac{n}{\ell^{t}}\right) q^{n}
\end{aligned}
$$

and similarly, making the change of variables $d \rightarrow d \ell^{t-1}$ gives us

$$
\sum_{n \geq 1}\left(\sum_{\substack{d \mid n \\ d>0}} d^{k-1} \delta\left(\frac{d}{\ell^{t-1}}\right)\right) q^{n}=\ell^{(t-1)(k-1)} \sum_{n \geq 1} \sigma_{k}\left(\frac{n}{\ell^{t-1}}\right) q^{n},
$$

where $\sigma_{k}\left(\frac{a}{b}\right)=0$ if $a$ and $b$ are integers with $b \neq 0$ but $\frac{a}{b} \notin \mathbb{Z}$. Substituting (28) and (29) in (27) yields

$$
E_{N, k, \chi}(z)=1-\frac{2 k}{B_{k}\left(\ell^{k}-1\right)} \sum_{n \geq 1}\left(\ell^{k} \sigma_{k-1}\left(\frac{n}{\ell^{t}}\right)-\sigma_{k-1}\left(\frac{n}{\ell^{t-1}}\right)\right) q^{n} .
$$

$E_{\ell^{t}, k, \chi}(z) \equiv 1(\bmod \ell)$ since $\operatorname{ord}_{\ell}\left(\frac{2 k}{B_{k}\left(\ell^{k}-1\right)}\right) \geq 1$ by Theorem 4.1. This proves Theorem 1.2.1.

Next, we assume $\ell=2$. If $\operatorname{ord}_{2}(k)=j$, then

$$
k=2^{j} m=\phi\left(2^{j+1}\right) m
$$


for some positive odd integer $m$. Moreover, Theorem 4.1 implies that $\operatorname{ord}_{2}\left(B_{k}\right)=$ -1 for every positive even integer $k \geq 4$. We examine $\operatorname{ord}_{2}\left(a\left(c_{N}\right)\right)$ in two cases.

CASE $1^{\prime} .2 \nmid N$.

$\operatorname{ord}_{2}\left(p^{k}-1\right) \geq j+1$ for every prime $p \mid N$ using (31), so

$$
\operatorname{ord}_{2}\left(a\left(c_{N}\right)\right) \leq 1+(j+1)(1-\omega(N))
$$

using (24). If $\omega(N)>1$, it follows that $E_{N, k, \chi}(z) \not \equiv 1(\bmod 2)$. Furthermore, if $N=p^{b}$ for some odd prime $p$ and positive integer $b$, and if $\operatorname{ord}_{2}\left(p^{k}-1\right)>j+1$, then $E_{p^{b}, k, \chi}(z) \not \equiv 1(\bmod 2)$.

CASE $2^{\prime} .2 \mid N$.

Using (31) and (24) as in case $1^{\prime}$, we obtain

$$
\operatorname{ord}_{2}\left(a\left(c_{N}\right)\right) \leq 1+(j+1)(2-\omega(N)) .
$$

If $\omega(N)>2$, then $E_{N, k, \chi}(z) \not \equiv 1(\bmod 2)$. Also, if $N=2^{a} p^{b}$ for some odd prime $p$ and positive integers $a$ and $b$, and if $\operatorname{ord}_{2}\left(p^{k}-1\right)>j+1$, then $E_{2^{a} p^{b}, k, \chi}(z) \not \equiv 1$ $(\bmod 2)$.

Hence, $E_{N, k, \chi}(z) \not \equiv 1(\bmod 2)$ if $N$ does not have the form $2^{a} p^{b}$, where $p$ is an odd prime satisfying

$$
\operatorname{ord}_{2}\left(p^{k}-1\right)=j+1
$$

and $a$ and $b$ are nonnegative integers. We therefore examine $E_{N, k, \chi}(z)(\bmod 2)$ when $N$ does have this form.

In the case where $N=2^{a}$ for some positive integer $a$ and in the case where $N=p^{b}$ where $p$ is an odd prime satisfying (32) and $b$ is a positive integer, the formulas for $E_{N, k, \chi}(z)$ are given by (30) with $\ell=2$ and $\ell=p$, respectively. The reasoning used there can also be used to show that $E_{N, k, \chi}(z) \equiv 1(\bmod 2)$ in these cases.

In the case where $N=2^{a} p^{b}$ for some odd prime $p$ satisfying (32) and $a$ and $b$ positive integers, a formula for $E_{2^{a} p^{b}, k, \chi}(z)$ can be found by applying the same reasoning used to derive (30):

$$
E_{2^{a} p^{b}, k, \chi}(z)=1-\frac{2 k}{B_{k}\left(p^{k}-1\right)\left(2^{k}-1\right)} \sum_{n \geq 1} A(n) q^{n},
$$

where

$$
\begin{aligned}
& A(n)= \\
& (2 p)^{k} \sigma_{k-1}\left(\frac{n}{2^{a} p^{b}}\right)-p^{k} \sigma_{k-1}\left(\frac{n}{2^{a-1} p^{b}}\right)-2^{k} \sigma_{k-1}\left(\frac{n}{2^{a} p^{b-1}}\right)+\sigma_{k-1}\left(\frac{n}{2^{a-1} p^{b-1}}\right) .
\end{aligned}
$$

Now observe that $E_{2^{a} p^{b}, k, \chi}(z) \equiv 1(\bmod 2) \operatorname{since} \operatorname{ord}_{2}\left(\frac{2 k}{B_{k}\left(p^{k}-1\right)\left(2^{k}-1\right)}\right)=1$ using Theorem 4.1. This completes the proof of Theorem 1.2. 


\section{The Proof of Theorem 1.3}

The proof of Theorem 1.3 follows by applying the Theorems of Carlitz (Theorems 5.1, 5.2) regarding the divisibility properties of the generalized Bernoulli numbers $B_{k, \chi}$ to the formula (4). We extend the definition of ord to rings of integers of number fields in the obvious way.

Theorem 5.1 (CARLitz) [C, Thm.1]. Suppose that $k$ is a positive integer, $\ell$ is a rational prime, and $\chi$ is a nontrivial primitive Dirichlet character with conductor $N$. Then

$$
\frac{B_{k, \chi}}{k}=\frac{\mathfrak{R}}{\mathfrak{D}},
$$

where $\mathfrak{R}$ and $\mathfrak{D}$ are elements in $O_{K_{\chi}}$ with $\operatorname{gcd}(\mathfrak{R}, \mathfrak{D})=1$. If $N$ has at least two distinct rational prime divisors, then $\mathfrak{D}=1$. If $N=\ell^{t}$, then $\mathfrak{D}$ is a product of prime divisors of $\ell$. that

We denote the series in formula (4) by $E_{N, k, \chi}(z)=\sum_{n \geq 0} b(n) q^{n}$, and observe

$$
b(1)=\frac{-2 k}{B_{k, \chi}} .
$$

If $N$ has at least two rational prime divisors, then there is an $\mathfrak{R} \in O_{K_{\chi}}$ for which $b(1)=\frac{-2}{\mathfrak{R}}$ by Theorem 5.1. It follows that if $\mathfrak{a}$ is an ideal in $O_{K_{\chi}, N}$ and $\mathfrak{a} \nmid(2)$, then $\operatorname{ord}_{\mathfrak{a}}(b(1)) \leq 0$, so that $E_{N, k, \chi}(z) \not \equiv 1(\bmod \mathfrak{a})$, proving Theorem 1.3.1. The proofs of Theorems 1.3.2-1.3.5 follow from Theorem 5.2.

Theorem 5.2 (CARlitz) [C, Thm.4]. Suppose $\chi$ is a nontrivial primitive character with conductor $N$, and $\ell$ is an odd rational prime. Then the following are true.

1. If $N=\ell$, then $\frac{B_{k, \chi}}{k} \in \mathbb{Z}$ unless there is a primitive root $g$ of $\mathbb{Z} / \ell \mathbb{Z}$ for which

$$
\mathfrak{p}=\operatorname{gcd}\left(\ell, 1-\chi(g) g^{k}\right) \neq(1),
$$

where $\mathfrak{p}$ is an ideal in $O_{K_{\chi}}$. In this case,

$$
\ell B_{k, \chi}+1 \equiv 0 \quad\left(\bmod \mathfrak{p}^{1+\operatorname{ord}_{\ell}(k)}\right) .
$$

2. If $N=\ell^{t}$ for some integer $t \geq 2$, then $\frac{B_{k, \chi}}{k} \in \mathbb{Z}$ unless there is a primitive root $g$ of $\mathbb{Z} / \ell \mathbb{Z}$ for which

$$
\mathfrak{p}=\operatorname{gcd}\left(\ell, 1-\chi(g) g^{k}\right) \neq(1),
$$

where $\mathfrak{p}$ is an ideal in $O_{K_{\chi}}$. In this case,

$$
(1-\chi(1+\ell)) \frac{B_{k, \chi}}{k} \equiv 1 \quad(\bmod \mathfrak{p}) .
$$

3. If $N=4$, then

$$
\frac{B_{k, \chi}}{k} \equiv \begin{cases}1\left(\bmod \frac{1}{2}\right) & \text { if } k \text { odd } \\ 0(\bmod 1) & \text { if } k \text { even }\end{cases}
$$

4. If $N=2^{t}$, for some integer $t \geq 3$, then $\frac{B_{k, x}}{k} \in \mathbb{Z}$. 
Note that if (33) holds, and if we let $B_{k, \chi}=\frac{U_{k, \chi}}{V_{k, \chi}}$, where $U_{k, \chi}$ and $V_{k, \chi} \in O_{K_{\chi}}$ and $\operatorname{gcd}\left(U_{k, \chi}, V_{k, \chi}\right)=1$, then

$$
\operatorname{ord}_{\mathfrak{p}}\left(B_{k, \chi}\right)=-\operatorname{ord}_{\mathfrak{p}}(\ell) \leq-1
$$

We now proceed with the proofs of Theorems 1.3.2-1.3.5. We assume in all cases that $\ell$ is an odd rational prime, $\mathfrak{a}$ is an ideal in $O_{K_{\chi}, N}$ with the property that $\mathfrak{a} \nmid(2), \chi$ is a nontrivial primitive Dirichlet character, and $k \geq 3$ is an integer satisfying $\chi(-1)=(-1)^{k}$.

Proof (of Theorem 1.3.2). If $N=4$ and $k$ is even, then $\chi$ is trivial. If $N=4$ and $k$ is odd, then $b(1)=\frac{-4}{s}$ for some nonzero integer $s$ by Theorem 5.2.3. If $\mathfrak{a} \nmid(4)$, then $\operatorname{ord}_{\mathfrak{a}}(b(1)) \leq 0$, so $E_{4, k, \chi}(z) \not \equiv 1(\bmod \mathfrak{a})$. If $N=2^{t}$ for some integer $t \geq 3$, then $b(1)=\frac{-2}{j}$ for some nonzero integer $j$ by Theorem 5.2.4. Hence, $\operatorname{ord}_{\mathfrak{a}}(b(1)) \leq 0$, so $E_{2^{t}, k, \chi}(z) \not \equiv 1(\bmod \mathfrak{a})$.

Proof (of Theorem 1.3.3). If $N=\ell$ and $\operatorname{gcd}\left(\ell, 1-\chi(g) g^{k}\right)=(1)$ for every primitive root $g$ of $\mathbb{Z} / \ell \mathbb{Z}$, then $b(1)=\frac{-2}{j}$ for some nonzero integer $j$ by Theorem 5.2.1. Hence, $\operatorname{ord}_{\mathfrak{a}}(b(1)) \leq 0$, so $E_{\ell, k, \chi}(z) \not \equiv 1(\bmod \mathfrak{a})$. If there is a primitive root $g$ of $\mathbb{Z} / \ell \mathbb{Z}$ for which $\mathfrak{p}=\operatorname{gcd}\left(\ell, 1-\chi(g) g^{k}\right) \neq(1)$, then $\operatorname{ord}_{\mathfrak{p}}\left(B_{k, \chi}\right)=-\operatorname{ord}_{\mathfrak{p}}(\ell) \leq-1$ by (34). It follows that $\operatorname{ord}_{\mathfrak{p}}(b(n)) \geq 1$ for every $n \geq 1$ by formula (4), and thus, $E_{\ell, k, \chi}(z) \equiv 1\left(\bmod \mathfrak{p}^{\operatorname{ord}_{\mathfrak{p}}(\ell)}\right)$.

Proof (of Theorem 1.3.4). Suppose $N=\ell$ and $\chi=(\dot{\bar{\ell}})$, the Legendre symbol. If we choose an arbitrary primitive root $g$ of $\mathbb{Z} / \ell \mathbb{Z}$ and suppose that there is an $a \in \mathbb{Z} / \ell \mathbb{Z}$ for which $a^{2} \equiv g(\bmod \ell)$, then $g^{\frac{\ell-1}{2}} \equiv a^{\ell-1} \equiv 1(\bmod \ell)$ since $\operatorname{gcd}(a, \ell)=1$. This contradicts the hypothesis that $g$ is a primitive root of $\mathbb{Z} / \ell \mathbb{Z}$, so $\left(\frac{g}{\ell}\right)=-1$. Using this fact, observe that $\operatorname{gcd}\left(\ell, 1-\left(\frac{g}{\ell}\right) g^{k}\right)=\operatorname{gcd}\left(\ell, 1+g^{k}\right) \neq(1)$ if and only if $g^{k} \equiv-1(\bmod \ell)$, i.e., if and only if $k \equiv \frac{\ell-1}{2}(\bmod \ell-1)$. In this case $\operatorname{gcd}\left(\ell, 1-\left(\frac{g}{\ell}\right) g^{k}\right)=(\ell)$, so $\operatorname{ord}_{\ell}\left(B_{k,(\dot{\bar{\ell}})}\right) \leq-1$ by (34). Using formula (4), it follows that $\operatorname{ord}_{\ell}(b(n)) \geq 1$ for all $n \geq 1$ if and only if $k \equiv \frac{\ell-1}{2}(\bmod \ell-1)$.

Proof (of Theorem 1.3.5). If $N=\ell^{t}$ for some integer $t \geq 2$, and if $\operatorname{gcd}(\ell, 1-$ $\left.\chi(g) g^{k}\right)=(1)$ for every primitive root $g$ of $\mathbb{Z} / \ell \mathbb{Z}$, then $b(1)=\frac{-2}{j}$ for some nonzero integer $j$ by Theorem 5.2.2. Hence, $\operatorname{ord}_{\mathfrak{a}}(b(1)) \leq 0$, so $E_{\ell^{t}, k, \chi}(z) \not \equiv 1(\bmod \mathfrak{a})$.

Q.E.D.

\section{References}

[A] T. Apostol, Introduction to Analytic Number Theory, Springer-Verlag, 1976.

[C] L. Carlitz, Arithmetic Properties of Generalized Bernoulli Numbers, J.Reine und Angew. Math. 202 (1959), 173-182.

[Ir-R] K. Ireland and M. Rosen, A Classical Introduction to Modern Number Theory, Springer-Verlag, New York, 1990 GTM 84.

[Iw] K. Iwasawa, Lectures on p-Adic L-Functions, Princeton Univ. Press, 1972.

[K] N. Koblitz, Introduction to Elliptic Curves and Modular Forms, Springer-Verlag, New York, 1984.

[Sc] B. Schoeneberg, Elliptic Modular Functions, Springer-Verlag New York Heidelberg Berlin, 1974. 
[Sw-D] H.P.F. Swinnerton-Dyer, On $\ell$-adic representations and congruences for coefficients of modular forms, Modular functions of one variable, Springer Lect. Notes in Math. 350 (1973), 1-55.

Department of Mathematics, University of Wisconsin, Madison, Wisconsin 53706

E-mail address: boylan@math.wisc.edu 